\title{
MODELAGEM DE MISTURAS DE TRÊS ESPÉCIES DE MADEIRAS NA FABRICAÇÃO DE CHAPAS AGLOMERADAS ESTRUTURAIS
}

\author{
Éverton Hillig ${ }^{1}$ \\ Clovis Roberto Haselein ${ }^{2}$ \\ Setsuo Iwakiri ${ }^{3}$
}

\begin{abstract}
RESUMO
Neste trabalho, foi analisada a viabilidade da utilização do planejamento centróide simplex para modelagem de experimentos com misturas de espécies de madeira na confecção de chapas aglomeradas. Para tanto, modelos lineares, quadráticos e cúbicos foram ajustados aos dados apresentados por Hillig (2000), para misturas de espécies de acácia negra, eucalipto e pinus e comparados ao modelo completo. As propriedades mecânicas e a densidade das chapas foram explicadas pelo modelo linear, enquanto que, para as propriedades de estabilidade dimensional, o modelo quadrático foi o mais adequado. A modelagem mostrou-se um método adequado de análise de misturas de espécies, permitindo reduzir o número de tratamentos com as mesmas conclusões obtidas utilizando o modelo completo.
\end{abstract}

\section{MODELING OF MIXTURES OF THREE WOOD SPECIES IN STRUCTURAL PARTICLEBOARD MANUFACTURING}

\begin{abstract}
The present work evaluated the use of simplex centroid design for modeling experiments of wood species mixture in particleboard manufacturing. In this regard, linear, quadratic and special cubic models were fitted to the data presented by Hillig (2000), for the mixtures of black battle, eucalypt and pine and compared to the full model. The mechanical properties and board density were explained by the linear model, while for the dimensional stability properties, the quadratic model was considered more adequate. The simplex design showed to be an adequate method of analyzing the mixture of species, allowing for a reduction in number of treatments with the same conclusions obtained with the full model.
\end{abstract}

\section{INTRODUÇÃO}

Estudos com misturas de espécies na confecção de chapas aglomeradas ou outros painéis são realizados com relativa freqüência. Nestes casos, para análise dos resultados, diversos métodos e técnicas estatísticas são utilizados dependendo, é claro, do número de espécies e outras variáveis envolvidas.

Vital (1973), estudando a produção de chapas aglomeradas com quatro espécies de madeira, puras ou misturadas, utilizou-se de técnicas de análise de regressão. Haselein
(1989), trabalhando com eucalipto e embaúba, também usou estas técnicas, sendo que, neste caso, considerou a proporção de uma delas como variável quantitativa de um experimento fatorial, tendo em vista as proporções de cada espécie serem complementares.

Em estudo realizado por Iwakiri et al. (1995), na mistura de diferentes proporções de Pinus elliottii $(0,42 \mathrm{~g} / \mathrm{cm} 3$ de massa específica) e Mimosa scabrella $(0,56 \mathrm{~g} / \mathrm{cm} 3)$, as misturas também foram consideradas como uma variável, ou seja, a proporção de uma delas. Del Menezzi et al (1996) confeccionaram

\footnotetext{
11 ehillig@ucs.br, Eng. Florestal, M.Sc, Professor Adjunto do Departamento de Engenharia e Informática, Universidade de Caxias do Sul, Av. João Dal Sasso, 800 - Bairro Universitário, CEP 95001-970, Bento Gonçalves (RS).

2 haseleic@ccr.ufsm.br, Eng. Florestal, PhD, Professor Adjunto do Departamento de Ciências Florestais, Centro de Ciências Rurais, Universidade Federal de Santa Maria, CEP 97015-900, Santa Maria (RS).

${ }^{3}$ setsuo@floresta.ufpr.br, Eng. Florestal, Dr., Professor Titular do Setor de Ciências Agrárias, Departamento de Engenharia e Tecnologia Florestal, Universidade Federal do Paraná, Av. Lothário Meissner, 3400, CEP 80210-170, Curitiba (PR).
} 
chapas com misturas de Pinus oocarpa $(0,46$ $\mathrm{g} / \mathrm{cm} 3)$ e Eucalyptus urophylla $(0,70 \mathrm{~g} / \mathrm{cm} 3)$, também considerando as misturas como proporções de uma das espécies.

Estes trabalhos têm em comum as conclusões de que as misturas, em geral, tendem a produzir painéis com maiores vantagens que a utilização de cada espécie individualmente e que a densidade básica das chapas tem grande influência sobre as propriedades mecânicas dos painéis, independente da espécie de madeira utilizada. Já para as propriedades de estabilidade dimensional, nota-se uma influência maior de fatores inerentes à espécie de madeira utilizada.

Para estudar o efeito da composição da mistura na variável resposta de um ensaio, pode-se utilizar a modelagem de misturas. Neste caso, duas considerações são importantes: as propriedades de uma mistura são determinadas pelas proporções de seus componentes e as proporções dos diversos componentes são dependentes entre si (Barros Neto et al, 2001). Assim, caso queira-se otimizar as propriedades de uma mistura mudando a sua formulação, deve-se sempre obedecer a regra de que a soma dos componentes tem de resultar em 100\%.

Experimentos que utilizam modelagem de misturas são freqüentemente realizados, principalmente nos setores de pesquisa química e biológica. Basicamente, este método utiliza-se da resolução de equações pelo método dos mínimos quadrados para encontrar soluções que expressem o comportamento de uma variável dependente em função da proporção de cada componente da mistura (Montgomery, 1997).

Em geral, para modelagem de misturas utilizam-se modelos lineares, quadráticos ou cúbicos que usam o mesmo número de ensaios que o número de coeficientes que se quer estimar. Este método recebeu nome próprio na literatura, sendo conhecido como "planejamento em rede simplex" e sendo utilizado principalmente para misturas de três componentes. Neste caso, devido à particularidade das misturas ternárias serem representadas por apenas uma mistura, o planejamento é conhecido como "centróide simplex".

Este trabalho tem por objetivo verificar a viabilidade de aplicação da modelagem de misturas em trabalhos com misturas de espécies para confecção de chapas aglomeradas, procurando determinar o melhor modelo a ser aplicado.

\section{MATERIAL E MÉTODOS}

\section{Universo de estudo}

Trabalhou-se com os dados do experimento desenvolvido em Hillig (2000), composto de 15 tratamentos constituídos de chapas aglomeradas estruturais com misturas de partículas de madeiras de Eucalyptus grandis W. Hill ex-Maiden, Pinus elliottii Engelm e Acacia mearnsii. Para confecção das chapas foram utilizadas partículas do tipo Flake, com dimensões nominais de $90 \times 20$ x $0,6 \mathrm{~mm}$, comprimento, largura e espessura, respectivamente. As partículas do tipo flake caracterizam-se por possuírem dimensões retangulares e são definidas como pequenas partículas de madeira com espessura uniforme, essencialmente planas e chatas, tendo a direção das fibras no plano das partículas, lembrando uma pequena peça de laminado (Carll, 1986).

Os tratamentos variaram conforme a participação de cada espécie na mistura, sendo realizadas três repetições que pressuporam uma restrição na casualização: a taxa de compressão. Utilizaram-se taxas de compressão de 1,21, 1,32 e 1,43, de forma a ampliar a abrangência de densidades de chapas fabricadas e permitir uma análise das misturas de espécies para cada taxa de compressão. Assim, foram produzidas 45 chapas aglomeradas do tipo estrutural, com 15 diferentes misturas de partículas de Pinus elliottii (15 anos de idade e $0,43 \mathrm{~g} / \mathrm{cm} 3 \mathrm{de}$ massa específica básica), Eucalyptus grandis (10 anos de idade e 0,47 g/cm3) e Acacia mearnsii (10 anos de idade e $0,64 \mathrm{~g} / \mathrm{cm} 3$ ).

A caracterização dos tratamentos para confecção das chapas, que denominou-se modelo completo, está apresentada na tabela 1. 
Tabela 1: Caracterização dos tratamentos para confecção das chapas - Modelo Completo

Table 1: $\quad$ Treatment characterization for board manufacturing - Complete Model

\begin{tabular}{ccccc}
\hline Tratamentos & Taxas de Compressão & \multicolumn{2}{c}{ Proporção de cada espécie (\%) } & Pcácia \\
\cline { 3 - 5 } & & Eucalipto & Pinus & 0 \\
\hline 1 & $1,21-1,32-1,43$ & 100 & 0 & 0 \\
\hline 3 & $1,21-1,32-1,43$ & 75 & 25 & 0 \\
\hline 4 & $1,21-1,32-1,43$ & 50 & 50 & 0 \\
\hline 5 & $1,21-1,32-1,43$ & 25 & 75 & 100 \\
\hline 6 & $1,21-1,32-1,43$ & 0 & 0 & 50 \\
\hline 7 & $1,21-1,32-1,43$ & 0 & 25 & 25 \\
\hline 8 & $1,21-1,32-1,43$ & 0 & 50 & 25 \\
\hline 9 & $1,21-1,32-1,43$ & 0 & 75 & 50 \\
\hline 10 & $1,21-1,32-1,43$ & 0 & 0 & 75 \\
\hline 11 & $1,21-1,32-1,43$ & 75 & 0 & 25 \\
\hline 12 & $1,21-1,32-1,43$ & 50 & 0 & 25 \\
\hline 13 & $1,21-1,32-1,43$ & 25 & 50 & 50 \\
\hline 14 & $1,21-1,32-1,43$ & 25 & 25 & 00 \\
\hline 15 & $1,21-1,32-1,43$ & 50 & 50 & 0 \\
\hline
\end{tabular}

${ }^{1}$ Proporção com base no peso seco total das partículas.

\section{Variáveis resposta}

Os testes realizados para avaliação da qualidade das chapas foram: absorção d'água e inchamento em espessura; flexão estática; arrancamento de pregos e ligação interna. Todos os testes seguiram as recomendações da norma norte-americana D 1037 - 93 (American Society for Testing and Materials, 1995). Os ensaios mecânicos foram feitos em Máquina Universal de Ensaios, hidráulica, marca Amsler, capacidade de 20 toneladas. Os pares de dados de carga e deformação foram alimentados automaticamente a uma placa de aquisição de dados, inserida em um computador IBM PC.

Além destas variáveis, conforme indicado na norma D 1037 - 93 (American Society for Testing and Materials, 1995), determinaram-se a massa específica aparente ou densidade aparente ( $\mathrm{Da}$ ) calculada pela razão entre a massa e o volume da amostra ao teor de umidade de equilíbrio e a massa específica básica ao teor de umidade de equilíbrio das chapas ou densidade básica ao teor de umidade de equilíbrio das chapas (Db) que foi calculada pela razão entre o peso seco em estufa e volume ao teor de umidade de equilíbrio de cada corpo de prova, condicionados a temperatura de $20 \mathrm{oC}$ e umidade relativa de $65 \%$.

\section{Modelagem das misturas}

O modelo mais simples para uma mistura é o modelo linear ou aditivo, que procura explicar o comportamento de uma propriedade apenas com os resultados obtidos com a utilização de cada componente individualmente, isto é, prever o comportamento de qualquer mistura sem realizar nenhuma mistura. Numa mistura de três componentes, pode-se ter, além deste, modelos quadráticos que consideram os efeitos das interações de dois componentes e modelos cúbicos que consideram os efeitos das interações dos três componentes.

Foram testados modelos cúbicos para todas as propriedades analisadas, visando determinar quais os coeficientes são significativos e, a partir deste ajuste, utilizar as 
misturas mínimas necessárias para estimar o modelo mais simples, cúbico, quadrático ou linear. Os modelos utilizados são expressos nas equações derivadas 1,2 e 3 , respectivamente.

$\mathrm{Yi}=\mathrm{b} 1 * \mathrm{X} 1+\mathrm{b} 2 * \mathrm{X} 2+\mathrm{b} 3 * \mathrm{X} 3$

$\mathrm{Yi}=\mathrm{b} 1 * \mathrm{X} 1+\mathrm{b} 2 * \mathrm{X} 2+\mathrm{b} 3 * \mathrm{X} 3+\mathrm{b} 12 * \mathrm{X} 1 * \mathrm{X} 2+$ $\mathrm{b} 13 * \mathrm{X} 1 * \mathrm{X} 3+\mathrm{b} 23 * \mathrm{X} 2 * \mathrm{X} 3$

$\mathrm{Yi}=\mathrm{b} 1 * \mathrm{X} 1+\mathrm{b} 2 * \mathrm{X} 2+\mathrm{b} 3 * \mathrm{X} 3+\mathrm{b} 12 * \mathrm{X} 1 * \mathrm{X} 2+$ $\mathrm{b} 13 * \mathrm{X} 1 * \mathrm{X} 3+\mathrm{b} 23 * \mathrm{X} 2 * \mathrm{X} 3+\mathrm{b} 123 * \mathrm{X} 1 * \mathrm{X} 2 * \mathrm{X} 3$
Onde:

$\mathrm{Y}_{\mathrm{i}}=$ Variável resposta

$\mathrm{b}_{\mathrm{i}}=$ Coeficientes

$\mathrm{Xi}=$ Proporção de cada espécie na mistura

Para determinação dos coeficientes foram utilizadas as sete misturas mínimas necessárias para o modelo cúbico. As misturas utilizadas neste trabalho para cada um dos modelos são apresentadas na tabela 2 .

Tabela 2: Tratamentos utilizados para cálculo dos coeficientes em cada modelo

Table 2: Treatments used for computing the coefficients in each model

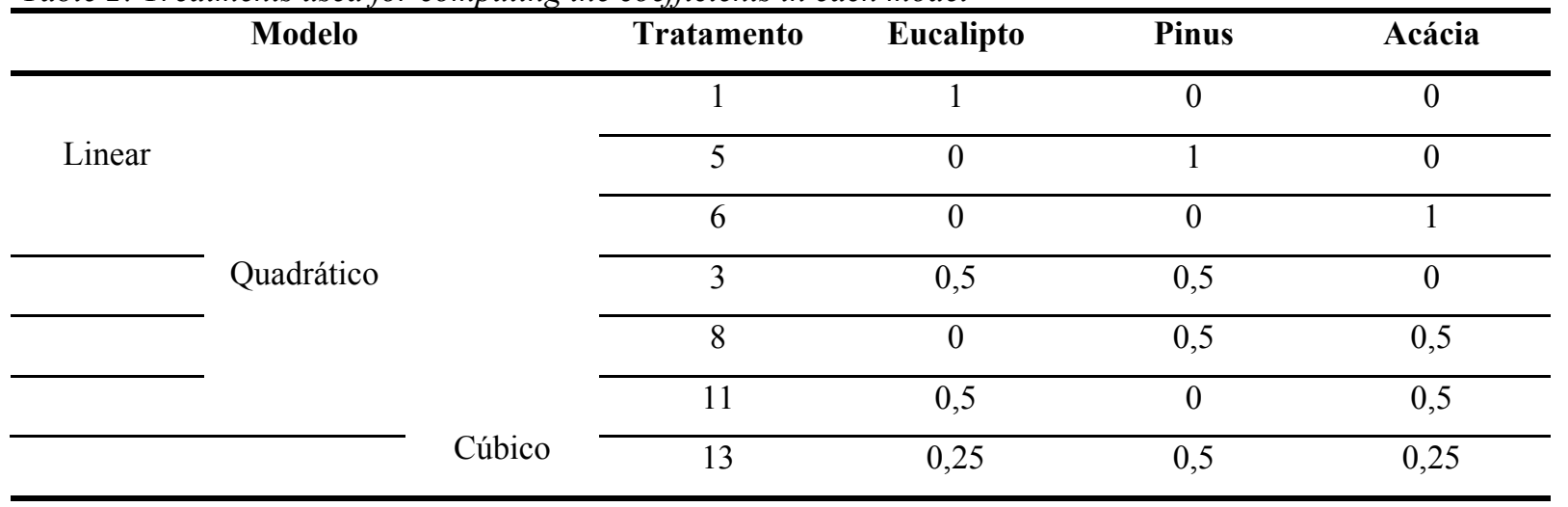

\section{Avaliação dos modelos}

$\mathrm{Na}$ avaliação dos modelos, três análises foram realizadas de forma a verificar sua aplicação. Primeiro foram testados modelos cúbicos para todas as propriedades analisadas. Foi realizada a análise da variância, e foram descartados os coeficientes não significativos quando comparados aos seus respectivos erros-padrão. Para derivação dos coeficientes, utilizou-se o software SPSS.

$\mathrm{Na}$ segunda etapa, realizou-se a modelagem com o número mínimo de misturas exigidas pelo modelo, e comparou-se a análise da variância da regressão deste modelo com a análise da variância obtida com o modelo cúbico.

Por fim, para os casos que o modelo mais simples (linear) não se apresentou suficiente para as estimativas, realizou-se a análise de variância das estimativas com uso dos dados do modelo completo visando comparar a eficiência dos modelos e comprovar a viabilidade de utilização do método.

\section{RESULTADOS E DISCUSSÃO}

\section{Propriedades mecânicas}

A tabela 3 apresenta os coeficientes dos modelos cúbicos calculados para cada uma das propriedades mecânicas e para a massa específica básica ou densidade básica das chapas ao teor de umidade de equilíbrio (Db), ajustados em função das sete misturas apresentadas na tabela 2 , juntamente com seus erros padrão e o valor de $\mathrm{F}$ calculado pela análise de variância. 
Tabela 3: Equações cúbicas para a densidade e propriedades mecânicas das chapas

Table 3: $\quad$ Cubic equations for density and mechanical properties of boards

\section{Erro Padrão}

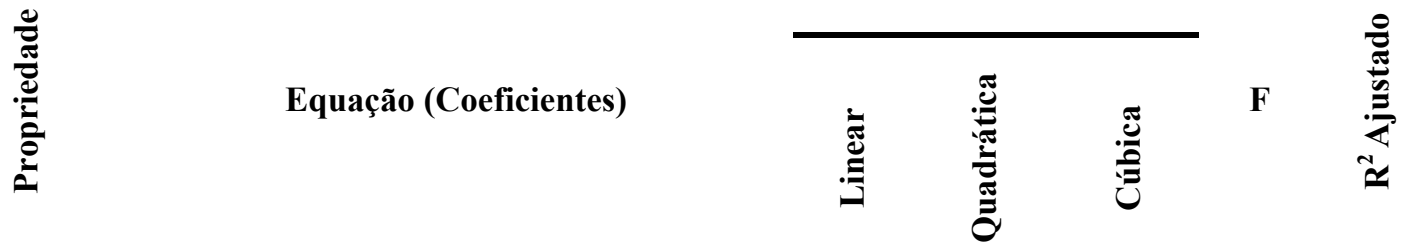

\begin{tabular}{|c|c|c|c|c|c|c|}
\hline $\mathrm{Db}$ & $\begin{array}{c}\mathrm{Db}=0,61 * \mathrm{PE}+0,67 * \mathrm{PP}+0,83 * \mathrm{PA}+ \\
0,052 * \mathrm{PE} * \mathrm{PP}+0,033 * \mathrm{PE} * \mathrm{PA}+ \\
0,067 * \mathrm{PP} * \mathrm{PA}+0,086 * \mathrm{PE}^{*} \mathrm{PP} * \mathrm{PA}\end{array}$ & 0,028 & 0,137 & 1,129 & $6,56^{\mathrm{s}}$ & 0,74 \\
\hline MOR & $\begin{array}{c}\mathrm{MOR}=227,2 * \mathrm{PE}+211,7 * \mathrm{PP}+349,1 * \mathrm{PA}+ \\
113,9 * \mathrm{PE}^{*} \mathrm{PP}-19,1 * \mathrm{PE}^{*} \mathrm{PA}+102,2 * \mathrm{PP} * \\
\mathrm{PA}+936,6^{*} \mathrm{PE}^{*} \mathrm{PP} * \mathrm{PA}\end{array}$ & 32,1 & 157,3 & 1296,9 & $2,33^{\mathrm{ns}}$ & 0,50 \\
\hline MOE & $\begin{array}{c}\mathrm{MOE}=34272 * \mathrm{PE}+31766 * \mathrm{PP}+52412 * \mathrm{PA} \\
\quad+14979 * \mathrm{PE} * \mathrm{PP}-12459 * \mathrm{PE} * \mathrm{PA}- \\
3133 * \mathrm{PP} * \mathrm{PA}+253500 * \mathrm{PE} * \mathrm{PP} * \mathrm{PA}\end{array}$ & 4597 & 225424 & 185745 & $2,39^{\mathrm{ns}}$ & 0,51 \\
\hline LI & $\begin{array}{c}\mathrm{LI}=1,40 * \mathrm{PE}+2,14 * \mathrm{PP}+2,07 * \mathrm{PA}+ \\
0,59 * \mathrm{PE} * \mathrm{PP}-0,75 * \mathrm{PE} * \mathrm{PA}-0,63 * \mathrm{PP} * \mathrm{PA}+ \\
3,14 * \mathrm{PE} * \mathrm{PP} * \mathrm{PA}\end{array}$ & 0,19 & 0,96 & 7,92 & $2,07^{\mathrm{ns}}$ & 0,46 \\
\hline Pregos & $\begin{array}{c}\text { Pregos }=22,8 * \mathrm{PE}+24,8 * \mathrm{PP}+20,3 * \mathrm{PA}-5,3 \\
* \mathrm{PE} * \mathrm{PP}-1,0 * \mathrm{PE} * \mathrm{PA}-3,7 * \mathrm{PP} * \mathrm{PA}- \\
22,0 * \mathrm{PE} * \mathrm{PP} * \mathrm{PA}\end{array}$ & 2,17 & 10,65 & 87,82 & $0,44^{\mathrm{ns}}$ & 0,16 \\
\hline
\end{tabular}

$\mathrm{Db}=$ Massa específica básica ao teor de umidade de equilíbrio ou Densidade básica das chapas ao teor de umidade de equilíbrio $\left(\mathrm{g} / \mathrm{cm}^{3}\right)$

$\mathrm{MOR}=$ Módulo de ruptura $\left(\mathrm{kgf} / \mathrm{cm}^{2}\right)$

MOE $=$ Módulo de elasticidade $\left(\mathrm{kgf} / \mathrm{cm}^{2}\right)$

$\mathrm{LI}=$ Ligação interna (tração perpendicular à superfície da chapa $-\mathrm{kgf} / \mathrm{cm}^{2}$ )

Pregos = Força necessária ao arrancamento de pregos $(\mathrm{kgf})$

$\mathrm{PE}=$ Proporção de eucalipto

$\mathrm{PP}=$ Proporção de pinus

PA = Proporção de acácia

${ }^{\mathrm{s}}$ Significativo ao nível de 5\% de probabilidade de erro.

${ }^{\text {ns }}$ Não significativo ao nível de $5 \%$ de probabilidade de erro.

Utilizando-se do cálculo de " $\mathrm{t}$ " para testar a significância dos coeficientes calculados, verifica-se que somente os coeficientes lineares são significativos, quando comparados aos seus erros-padrão, mostrando que cada espécie tem participação nos resultados encontrados em todas as propriedades analisadas, porém não ocorreram interações entre espécies.

Também, as equações não apresentaram significância estatística, pelo teste de "F", com exceção da equação para Db. No entanto, o número de parâmetros utilizados foi demasiado, pois o modelo linear pode ser ajustado apenas considerando as chapas confeccionadas com cada espécie pura.
Visando comprovar estas afirmações, foram realizadas as mesmas análises anteriores considerando apenas os dados encontrados para as chapas confeccionadas com cada espécie pura. Os resultados são apresentados na tabela 4. Nota-se que os coeficientes lineares apresentaram os mesmos valores, pois os dados que os deram origem são os mesmos. Por outro lado, os erros padrão dos coeficientes apresentaram valores semelhantes e os valores de R2 aumentaram em todos os casos. O teste "F", por sua vez, mostrou que somente para arrancamento de pregos a regressão foi não significativa, indicando que apenas as chapas puras conseguem expressar o comportamento das misturas para qualquer mistura nas outras propriedades analisadas. 
Tabela 4: Equações lineares para a densidade e propriedades mecânicas das chapas, utilizando-se apenas dos dados das chapas confeccionadas com cada espécie pura

Table 4: Linear equations for density and mechanical properties of boards, using only data of boards manufactured with each one species used single

\begin{tabular}{ccccc}
\hline Prop. & Equação & Erro padrão & $\mathrm{F}$ & $\mathrm{R}^{2}$ \\
\hline $\mathrm{Db}$ & $\mathrm{Dens}=0,61 * \mathrm{PE}+0,67 * \mathrm{PP}+0,83 * \mathrm{PA}$ & 0,022 & $24,6^{\mathrm{s}}$ & 0,89 \\
$\mathrm{MOR}$ & $\mathrm{MOR}=227,2 * \mathrm{PE}+211,7 * \mathrm{PP}+349,11 * \mathrm{PA}$ & 27,1 & $7,7^{\mathrm{s}}$ & 0,72 \\
$\mathrm{MOE}$ & $\mathrm{MOE}=34.272 * \mathrm{PE}+31.765^{*} \mathrm{PP}+52.412 * \mathrm{PA}$ & 4.870 & $5,4^{\mathrm{s}}$ & 0,64 \\
$\mathrm{LI}$ & $\mathrm{LI}=1,40 * \mathrm{PE}+2,14 * \mathrm{PP}+2,07 * \mathrm{PA}$ & 0,19 & $5,1^{\mathrm{s}}$ & 0,61 \\
Pregos & Pregos $=22,8 * \mathrm{PE}+24,8 * \mathrm{PP}+20,3 * \mathrm{PA}$ & 1,53 & $2,2^{\mathrm{ns}}$ & 0,42 \\
\hline
\end{tabular}

${ }^{\mathrm{s}}$ Significativo ao nível de $5 \%$ de probabilidade de erro.

${ }^{\text {ns }}$ Não significativo ao nível de $5 \%$ de probabilidade de erro.

Outras análises podem ser realizadas utilizando-se dos dados obtidos com as espécies puras, como, por exemplo, as correlações existentes entre as variáveis e o ajuste de modelos das propriedades físicomecânicas das chapas em função de outras variáveis.
Nas Figuras 1 e 2 pode-se observar as estimativas de MOR e MOE em função da densidade, destacando o coeficiente de determinação de 0,70 para MOR e de 0,63 para MOE.

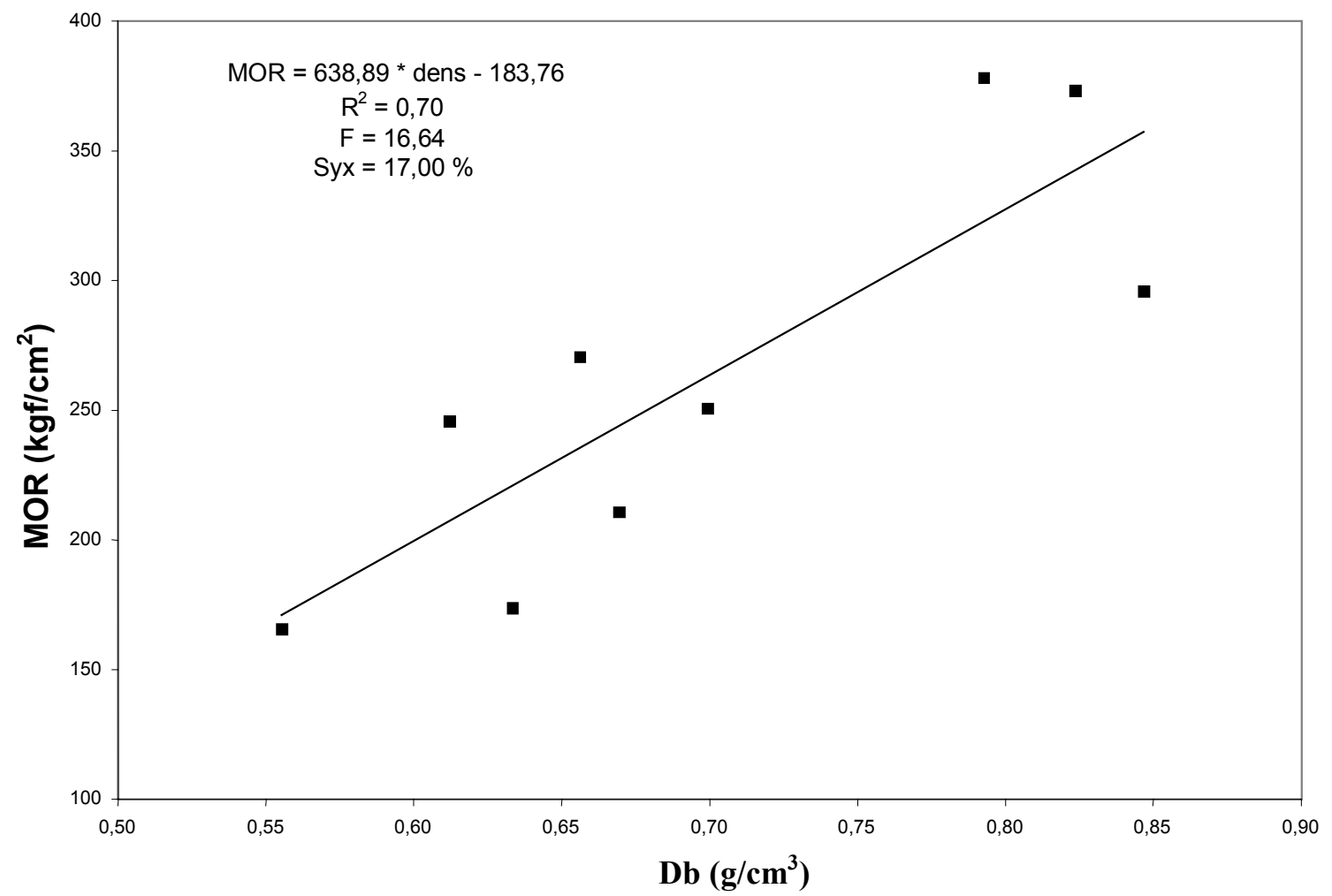

Figura 1: Gráfico de MOR em função da massa específica básica ao teor de umidade de equilíbrio ou densidade básica da chapa ao teor de umidade de equilíbrio $(\mathrm{Db})$, estimado com os valores obtidos com as espécies puras

Figure 1: Graph of MOR as a function of density of board at equilibrium moisture content (Db), estimated using the values obtained with each one species 


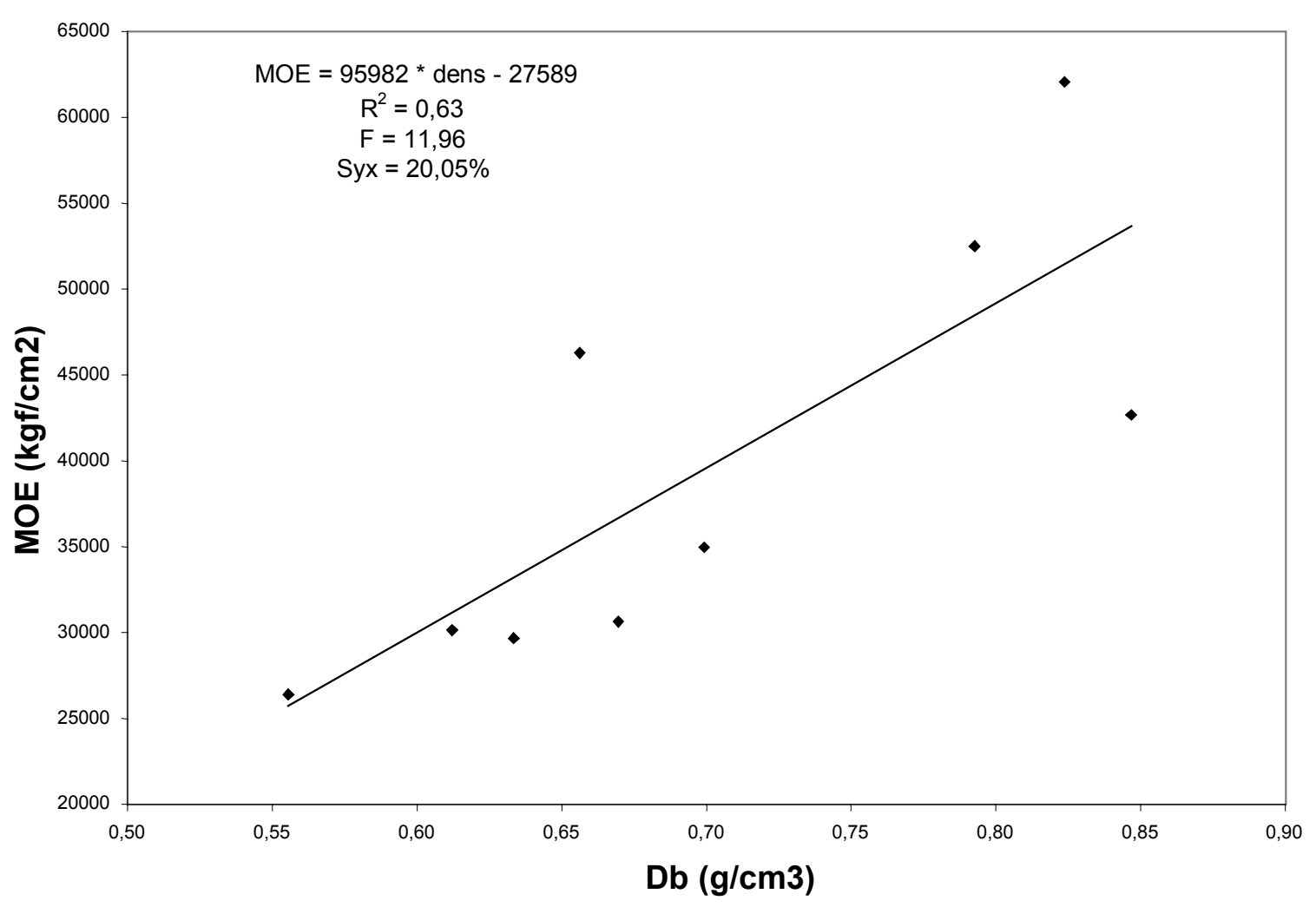

Figura 2: Gráfico de MOE em função da massa específica básica ao teor de umidade de equilíbrio ou densidade básica da chapa ao teor de umidade de equilíbrio (Db), estimado com os valores obtidos com as espécies puras

Figure 2: Graph of MOE as a function of density of board at equilibrium moisture content (Db), estimated using the values obtained with each one species

Os gráficos apresentados nas Figuras 1 e 2 são semelhantes aos apresentados em Hillig (2000), que se utilizou dos dados das 15 misturas, caracterizadas como modelo completo, para as estimativas. Este fato comprova a tese de que as misturas mínimas utilizadas na modelagem de misturas permitem as mesmas análises que um modelo que se utilize de mais ensaios.

\section{Estabilidade dimensional}

A tabela 5 apresenta os coeficientes calculados para o modelo cúbico, com sete misturas, juntamente com os dados complementares da análise de variância, para as propriedades de estabilidade dimensional das chapas. 
Tabela 5: Equações cúbicas para a densidade e propriedades mecânicas das chapas Table 5: Cubic equation for density and mechanical properties of boards

\begin{tabular}{|c|c|c|c|c|c|c|}
\hline \multirow{2}{*}{ 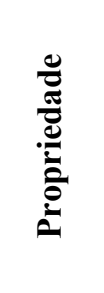 } & \multirow[b]{2}{*}{ Equação (Coeficientes) } & \multicolumn{3}{|c|}{ Erro Padrão } & \multirow[b]{2}{*}{$\mathbf{F}$} & \multirow[b]{2}{*}{$\mathbf{R}^{2}$} \\
\hline & & $\stackrel{\leftrightarrows}{\leftrightarrows}$ & : & 先 & & \\
\hline AP2 & $\begin{array}{c}\mathrm{AP} 2=7,87 * \mathrm{PE}+15,71 * \mathrm{PP}+6,50 * \mathrm{PA}- \\
10,61 * \mathrm{PE} * \mathrm{PP}+0,31 * \mathrm{PE} * \mathrm{PA}+7,22 * \mathrm{PP} * \mathrm{PA}- \\
19,48 * \mathrm{PE}^{*} \mathrm{PP} * \mathrm{PA}\end{array}$ & 0,90 & 4,43 & 36,53 & $11,32^{\mathrm{s}}$ & 0,83 \\
\hline AP24 & $\begin{array}{c}\mathrm{AP} 24=41,64 * \mathrm{PE}+70,64 * \mathrm{PP}+24,80 * \mathrm{PA}- \\
46,30 * \mathrm{PE} * \mathrm{PP}-11,61 * \mathrm{PE} * \mathrm{PA}-37,12 * \mathrm{PP} * \mathrm{PA}- \\
162,95 * \mathrm{PE} * \mathrm{PP} * \mathrm{PA}\end{array}$ & 3,55 & 17,41 & 143,5 & $17,19^{\mathrm{s}}$ & 0,88 \\
\hline AV2 & $\begin{array}{c}\mathrm{AV} 2=5,05 * \mathrm{PE}+11,01 * \mathrm{PP}+5,57 * \mathrm{PA}- \\
6,88 * \mathrm{PE} * \mathrm{PP}+1,56 * \mathrm{PE} * \mathrm{PA}-3,21 * \mathrm{PP} * \mathrm{PA}- \\
12,12 * \mathrm{PE} * \mathrm{PP} * \mathrm{PA} \\
\end{array}$ & 0,45 & 2,20 & 18,12 & $20,27^{\mathrm{s}}$ & 0,90 \\
\hline AV24 & $\begin{array}{c}\mathrm{AV} 24=26,87 * \mathrm{PE}+49,72 * \mathrm{PP}+21,26 * \mathrm{PA}- \\
29,25^{*} \mathrm{PE} * \mathrm{PP}-1,72 * \mathrm{PE} * \mathrm{PA}-19,12 * \mathrm{PP} * \mathrm{PA}- \\
103,8 \mathrm{P}^{*} \mathrm{PE} * \mathrm{PP} * \mathrm{PA}\end{array}$ & 1,92 & 9,42 & 77,69 & $23,59^{\mathrm{s}}$ & 0,91 \\
\hline IE2 & $\begin{array}{c}\mathrm{IE} 2=3,28 * \mathrm{PE}+4,81 * \mathrm{PP}+2,64 * \mathrm{PA}-0,89 \\
* \mathrm{PE} * \mathrm{PP}+3,02 * \mathrm{PE} * \mathrm{PA}+0,08 * \mathrm{PP} * \mathrm{PA}+ \\
9,26 * \mathrm{PE} * \mathrm{PP} * \mathrm{PA}\end{array}$ & 0,39 & 1,92 & 15,87 & $3,10^{\mathrm{s}}$ & 0,57 \\
\hline IE24 & $\begin{array}{c}\mathrm{IE} 24=15,58 * \mathrm{PE}+25,30 * \mathrm{PP}+10,08 * \mathrm{PA}-7,76 \\
* \mathrm{PE} * \mathrm{PP}+3,75 * \mathrm{PE} * \mathrm{PA}+2,25 * \mathrm{PP} * \mathrm{PA}- \\
12,56 * \mathrm{PE} * \mathrm{PP} * \mathrm{PA}\end{array}$ & 1,28 & 6,25 & 51,56 & $13,75^{\mathrm{s}}$ & 0,85 \\
\hline
\end{tabular}

AP2 = Absorção em peso para 2 horas de imersão em água (\%).

AP24 = Absorção em peso para 24 horas de imersão em água (\%).

AV2 = Absorção em volume para 2 horas de imersão em água (\%).

AV24 = Absorção em volume para 24 horas de imersão em água (\%).

IE2 = Inchamento em espessura após 2 horas de imersão em água (\%).

IE24 = Inchamento em espessura após 24 horas de imersão em água (\%).

$\mathrm{PE}=$ Proporção de eucalipto

$\mathrm{PP}=$ Proporção de pinus

PA = Proporção de acácia

${ }^{\mathrm{s}}$ Significativo ao nível de $5 \%$ de probabilidade de erro

Da mesma forma que para as propriedades mecânicas, utilizou-se do teste " $\mathrm{t}$ " para determinar os coeficientes significativos, em comparação com seus respectivos erros-padrão. Também neste caso, os coeficientes de maior influência nas propriedades das chapas foram os das espécies puras. Por outro lado, neste caso observa-se também que ocorreu certa interação entre as misturas binárias, principalmente para as misturas de eucalipto com pinus e pinus com acácia. Assim, foram descartados os coeficientes não significativos e obteve-se uma equação simplificada para cada uma das propriedades analisadas, que são apresentadas nas equações 4 a 9 .

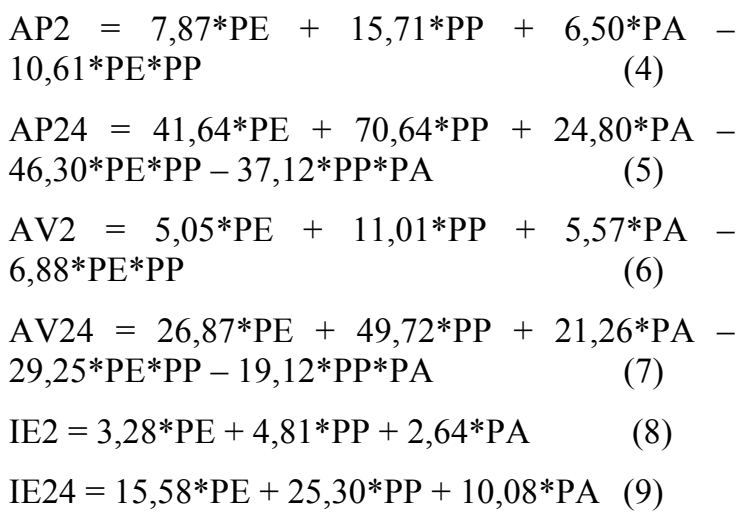

$\mathrm{IE} 2=3,28 * \mathrm{PE}+4,81 * \mathrm{PP}+2,64 * \mathrm{PA}$

$\mathrm{IE} 24=15,58 * \mathrm{PE}+25,30 * \mathrm{PP}+10,08 * \mathrm{PA}$

Nas equações simplificadas 4 a 9 podese observar que para AP e AV houve influência das misturas binárias, entre eucalipto e pinus para AP2 e AV2, e entre 
eucalipto e pinus e pinus e acácia para AP24 e AV24. Também, em função de todos os coeficientes serem negativos pode-se dizer que as espécies interagiram antagonisticamente. Como neste caso o que se quer é uma menor taxa de cada uma das propriedades analisadas, pode-se dizer então que a mistura destas espécies traz um efeito benéfico para estas propriedades, adicional ao efeito da soma individual de cada uma.

Outras observações podem ser tiradas pela análise das equações desenvolvidas como, por exemplo, que a espécie pinus apresenta maior influência sobre todas as propriedades de estabilidade dimensional, pois os coeficientes para esta espécie foram maiores em todos os casos.

Tabela 6; Análise de Variância para AP e AV do ajuste dos modelos linear e quadrático aos valores encontrados com as 15 misturas com 3 repetições

Table 6: Analysis of variance for AP and AV of the fitted linear and quadratic models at the values found with the 15 mixtures with three replications

\begin{tabular}{|c|c|c|c|c|c|}
\hline Propriedade & Modelo & FV & GL & SQ & $\mathbf{Q M}$ \\
\hline \multirow{6}{*}{ AP2 } & \multirow{3}{*}{ Linear } & Regressão & 2 & 204,94 & 102,47 \\
\hline & & Resíduo & 42 & 96,68 & 2,30 \\
\hline & & Falta de ajuste & 12 & 29,17 & 2,43 \\
\hline & \multirow{3}{*}{ Quadrático } & Regressão & 5 & 230,96 & 46,19 \\
\hline & & Resíduo & 39 & 70,66 & 1,81 \\
\hline & & Falta de ajuste & 9 & 3,15 & 0,35 \\
\hline \multirow{6}{*}{ AP24 } & \multirow{3}{*}{ Linear } & Regressão & 2 & $5.362,81$ & $2.681,40$ \\
\hline & & Resíduo & 42 & $2.207,20$ & 52,55 \\
\hline & & Falta de ajuste & 12 & 490,24 & 40,85 \\
\hline & \multirow{3}{*}{ Quadrático } & Regressão & 5 & $5.840,22$ & $1.168,04$ \\
\hline & & Resíduo & 39 & $1.729,79$ & 44,35 \\
\hline & & Falta de ajuste & 9 & 12,83 & 1,43 \\
\hline \multirow{6}{*}{ AV2 } & \multirow{3}{*}{ Linear } & Regressão & 2 & 83,23 & 41,61 \\
\hline & & Resíduo & 42 & 34,55 & 0,82 \\
\hline & & Falta de ajuste & 12 & 14,82 & 1,23 \\
\hline & \multirow{3}{*}{ Quadrático } & Regressão & 5 & 96,97 & 19,39 \\
\hline & & Resíduo & 39 & 20,81 & 0,53 \\
\hline & & Falta de ajuste & 9 & 1,08 & 0,12 \\
\hline \multirow{6}{*}{ AV24 } & \multirow{3}{*}{ Linear } & Regressão & 2 & $1.892,49$ & 946,24 \\
\hline & & Resíduo & 42 & 828,66 & 19,73 \\
\hline & & Falta de ajuste & 12 & 261,05 & 21,75 \\
\hline & \multirow{3}{*}{ Quadrático } & Regressão & 5 & $2.168,24$ & 433,65 \\
\hline & & Resíduo & 39 & 552,91 & 14,18 \\
\hline & & Falta de ajuste & 9 & 14,7 & 1,63 \\
\hline
\end{tabular}

O resumo da análise da variância apresentado na tabela 6 permite verificar a aplicação do método de modelagem para o estudo em questão e a comparação dos modelos utilizados, linear e quadrático. Para as propriedades apresentadas, a inclusão das misturas que não foram utilizadas na modelagem confirmam a aplicação do método,
Para comparar a eficiência dos modelos linear e quadrático nas estimativas de AP e AV e também testar a qualidade dos modelos desenvolvidos, realizou-se as estimativas para estas variáveis utilizando-se dos dados do modelo completo (15 misturas com 3 repetições). Estas estimativas visaram confirmar a aplicação do método por meio da utilização de misturas que não foram usadas na modelagem e permitiram a análise de variância com a inclusão da falta de ajuste para os resultados obtidos. A tabela 6 apresenta o resumo da análise de variância para a absorção em peso e absorção em volume, comparando os modelos linear e quadrático, com a utilização dos dados do modelo completo. 


\section{CONCLUSÕES}

A modelagem de misturas mostrou-se um método viável para aplicação em experimentos com misturas de espécies para confecção de chapas aglomeradas estruturais. Neste caso, apenas os seis tratamentos utilizados para o modelo quadrático permitem as mesmas conclusões que os quinze tratamentos do modelo completo.

As propriedades mecânicas das chapas e sua massa específica, ou densidade, foram explicadas pelo modelo linear, enquanto para as propriedades de estabilidade dimensional o modelo quadrático apresentou ganhos nas estimativas. Desta forma, ensaios com misturas de espécies para confecção de chapas aglomeradas estruturais podem ser realizados com menor número de tratamentos e maior número de repetições, visando diminuir o erro experimental e melhorar a qualidade do trabalho. Recomenda-se o uso da modelagem de misturas com modelos lineares para as propriedades mecânicas e modelos quadráticos para as propriedades de estabilidade dimensional.

\section{REFERÊNCIAS}

AMERICAN SOCIETY FOR TESTING AND MATERIALS. Standard test methods for evaluating properties of wood-base fiber and particle panel materials, D 1037 - 93. Philadelphia, PA. 1995.

BARROS NETO, B., SCARMINIO, I.S., BRUNS, R. E. Como fazer experimentos Pesquisa e desenvolvimento na ciência e na indústria. Campinas, SP: Editora da Unicamp, 2001. $401 \mathrm{p}$.

CARLL, C. Wood particleboard and flake board: Types, grades, and uses. General Technical Report FPL-GTR-53. Madison, WI: U. S. Department of Agriculture, Forest Service, Forest Products Laboratory, 1986. 9 p.

DEL MENEZZI, C. H. S., SOUZA, M. R., GONÇALEZ, J. C. Fabricação e avaliação tecnológica da chapa aglomerada de mistura de Eucalyptus urophylla T. S. Blake e Pinus oocarpa Schiede. Revista Árvore. v. 20, n. 3, p. 371-379, 1996.
HASELEIN, C. R. Análise de parâmetros para a fabricação de chapas de partículas aglomeradas de madeira de eucalipto (Eucalyptus grandis W. Hill ex- Maiden) e embaúba (Cecropia sp.). Viçosa, 1989. 75 p. Dissertação (Mestrado em Ciência Florestal) Universidade Federal de Viçosa.

HILLIG, E. Qualidade de chapas aglomeradas estruturais, fabricadas com madeiras de pinus, eucalipto e acácia negra, puras ou misturadas, coladas com taninoformaldeído. Santa Maria, 2000. 96 p. Dissertação (Mestrado em Ciência Florestal) Universidade Federal de Santa Maria.

IWAKIRI, S., KLOCK, U., ROCHA, M. P. et al. Mistura de espécies na produção de chapas de partículas estruturais "waferboard". Agrárias, v. 14, n. 1/2, p. 107-114, 1995.

MONTGOMERY, D. C. Design and analysis of experiments. 4. ed. New York: John Wiley \& Sons, 1997. $704 \mathrm{p}$.

VITAL, B. R. Effects of species and panel densities on properties of hardwood particleboard. Madison, 1973. 111 p. Thesis (Master of Science - Forestry) - University of Wisconsin. 